\title{
75\% Enhancement of the Second Order Nonlinearity in Twin-Hole Fibres through Voltage Assisted Cooling during Poling
}

\author{
Albert Canagasabey, Francesco P. Mezzapesa, Costantino Corbari, Peter G. Kazansky, Morten Ibsen \\ Optoelectronics Research Centre, University of Southampton, S017 1BJ, United Kingdom
}

A technique by which the second order nonlinearity of poled silica fibre is significantly increased is demonstrated. Voltage assisted cooling technique offers a significant increase in $\chi^{(2)}$ compared to the standard thermal poling technique.

Amorphous silica does not posses an intrinsic second order nonlinearity (SON) due to its centrosymmetric nature. However, in the process of thermal poling, the intrinsic third order nonlinearity of silica combines with the frozen in-field created by thermal poling to produce an effective SON, with values as high as $1 \mathrm{pm} / \mathrm{V}$ reported in the literature for bulk silica and silica fibres [1,2]. However, high values of SON have not been consistently achieved in twin-hole fibres as the electro-optic effect induced is affected by many factors, such as fibre geometry and core composition. The average value of SON is more likely to be in the region of $0.1 \mathrm{pm} / \mathrm{V}$ for poled silica fibres. Poled silica fibres can be utilised to fabricate devices such as, electro-optic modulators, optical switches, frequency converters, optical parametric oscillators, etc.

The technique of voltage assisted cooling involves gradual incremental increase of the applied DC field whilst the temperature is gradually reduced [3]. Thus the applied DC voltage is maintained close to the breakdown voltage of silica, which increases as this is being cooled as a result of lowered mobility of charges. This technique, when applied to bulk Herasil samples, showed a 75\% increase in the second harmonic power [3]. In that report, the depth of the second order nonlinear region (or depletion region) was also observed to increase significantly for samples poled using the voltage assisted cooling technique.

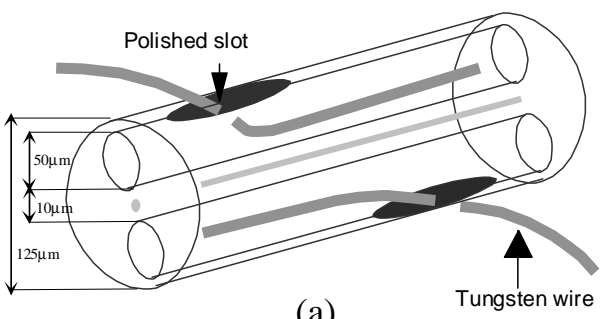

(a)

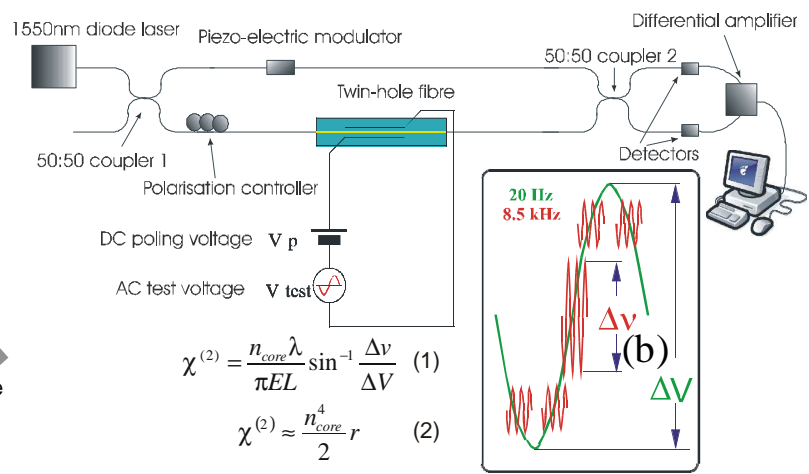

Figure 1(a). A $125 \mu \mathrm{m}$ diameter twin-hole fibre device with two $25 \mu \mathrm{m}$ gold coated tungsten electrodes. (b). All-fibre Mach-Zehnder interferometer for the in-situ measurement of the SON during thermal poling of the twin-hole fibre device. Equation 1 shows the calculation of the SON susceptibility, $\chi^{(2)}$ for a device of length, $\mathrm{L}$ and applied electric field, $\mathrm{E}$ from the amplitudes of the two modulation frequencies. Equation 2 shows the relationship between $\chi^{(2)}$ and linear electro-optic coefficient, $r$, through the core refractive index, $n_{\text {core }}$.

In this experiment, twin-hole fibre of NA 0.28 and germanium concentration of $16.8 \mathrm{~mol} \%$ was used to fabricate devices with two side entry electrodes, as shown in Figure 1(a).One of the holes is positioned closer to the core to attain better overlap with the depletion region, which in fibres typically is $\sim 3-5 \mu \mathrm{m}$ wide [4]. The devices were fusion spliced into one arm of an all-fibre Mach Zehnder interferometer for the in-situ measurement of the linear electro-optic (LEO) coefficient during the poling process. This is schematically illustrated in Figure 1(b). 
The evolution of the LEO coefficient for the two devices as measured by the interferometric technique described above is shown in Figure 2. Device 1 was poled at a temperature of $290^{\circ} \mathrm{C}$ and a constant $3 \mathrm{kV}$ DC voltage for $\sim 23$ minutes, while Device 2 was poled at the same conditions for $\sim 22$ minutes before the temperature was reduced to $265^{\circ} \mathrm{C}$ and the voltage increased to $3.5 \mathrm{kV}$. The voltage was again increased to $4 \mathrm{kV}$ and $4.5 \mathrm{kV}$ for the poling temperatures $240^{\circ} \mathrm{C}$ and $220^{\circ} \mathrm{C}$ respectively. The dwell time of the applied field at each temperature is not critical and does not result in a greater residual SON, as verified in subsequent experiments. It was also observed that extended periods of poling at any of the temperature/voltage combinations can result in an electrical breakdown across the core of the fibre.

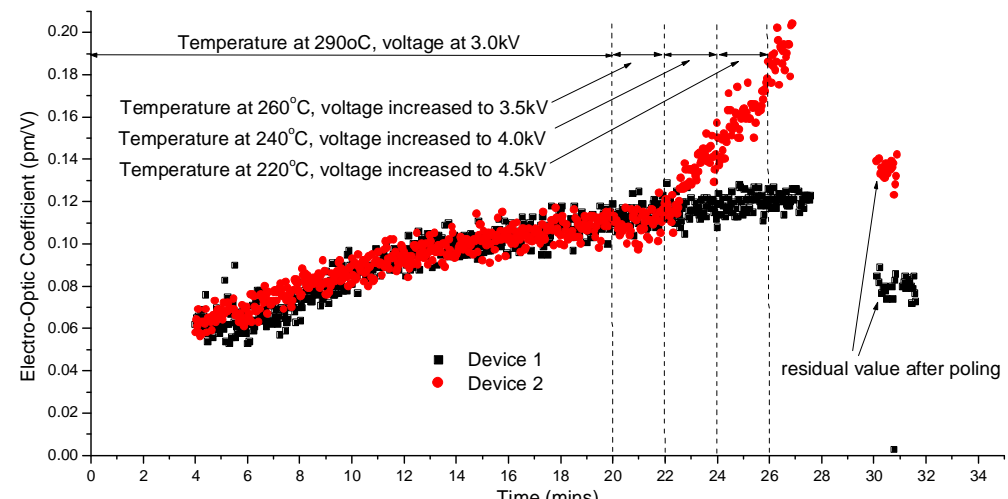

Figure 2. Evolution of the electro-optic coefficient during thermal poling of twin-hole fibre devices. The initial parts of the curves showing rise and fall of the E-O coefficient during the first few minutes of poling have been omitted for clarity. The residual values of the LEO were measured with the DC field switched off, as represented by the drop seen from the maximum LEO attained at the end of poling.

The residual values of the LEO coefficient, which is measured after the high voltage DC is switched off, were 0.08 and $0.14 \mathrm{pm} / \mathrm{V}$ for devices 1 and 2 , respectively. These values were recorded immediately after the poling procedures. The drop seen in the measured LEO coefficient in Figure 2 is due to the removal of the applied DC field component. The difference in the measured values of LEO coefficient represents a $75 \%$ increase in the induced electro-optic effect for the device poled with the voltage assisted cooling technique over the standard poling technique. The significant improvement in the SON in Device 2 with voltage assisted cooling is attributed to the increased injection of the less mobile carriers $\left(\mathrm{H}^{+}, \mathrm{K}^{+} \ldots\right)$ at the anode surface in the presence of a stronger electric field, resulting in a greater frozen-in field after poling. The increase in the concentration of the injected carriers resulted in a broader nonlinear layer in the case of the bulk Herasil samples [3]. In the case of poled fibres, a broader nonlinear layer could result in a more uniform distribution of charges and consequently a more uniform $\mathrm{SON}$ in the core of the fibre.

In conclusion, voltage-assisted cooling during poling of twin-hole fibres significantly enhances second order nonlinearity compared to the standard poling technique. Furthermore, the width of the nonlinear layer is possibly broader for fibres poled using the voltage assisted cooling technique. This suggests that the positioning of the core of the fibre near the anode hole may no longer be critical as in standard poling technique [5]. If the fibre is manufactured with the core positioned away from the anode hole and equidistant from the two holes, the current transmission losses due to leakage into the anode hole can be improved. This could serve to further improve the conversion efficiency of second harmonic generation in periodically poled silica fibres [5].

1. Myers, R.A., N. Mukerjee, and S.R.J. Brueck, "Large second-order nonlinearities in poled fused silica",. Optics Letters, 1991. 16(22): p. 1732-1734.

2. Kazansky, P.G., L. Dong, and P.St.J. Russell, "High second-order nonlinearities in poles silicate fibres", Optics Letters, 1994. 19(10): p. 701-703.

3. $\quad$ Mezzapesa, F.P., et al., "Voltage-assisted cooling: a new route to enhance $\chi^{(2)}$ during thermal poling", CLEO/QELS, May 2005 CMW7, Baltimore, MD, USA

4. An, H., and S. Fleming, "Investigation of the spatial distribution of second-order nonlinearity in thermally poled optical fibres" Optics Express, 2005, 13(9): p 3500-3005.

5. Corbari, C., et al., "All-fibre frequency conversion in long periodically poled silica fibres", OFC, March 2005, OFB3, Anaheim, CA, USA 